\title{
Local Delivery of Glial Cell Line-Derived Neurotrophic Factor Improves Facial Nerve Regeneration After Late Repair
}

\author{
Florian M. Barras, MD; Thierry Kuntzer, MD; Anne D. Zurn, PhD; Philippe Pasche, MD
}

Objectives/Hypothesis: Facial nerve regeneration is limited in some clinical situations: in long grafts, by aged patients, and when the delay between nerve lesion and repair is prolonged. This deficient regeneration is due to the limited number of regenerating nerve fibers, their immaturity and the unresponsiveness of Schwann cells after a long period of denervation. This study proposes to apply glial cell line-derived neurotrophic factor (GDNF) on facial nerve grafts via nerve guidance channels to improve the regeneration.

Methods: Two situations were evaluated: immediate and delayed grafts (repair 7 months after the lesion). Each group contained three subgroups: a) graft without channel, b) graft with a channel without neurotrophic factor; and c) graft with a GDNFreleasing channel. A functional analysis was performed with clinical observation of facial nerve function, and nerve conduction study at 6 weeks. Histological analysis was performed with the count of number of myelinated fibers within the graft, and distally to the graft. Central evaluation was assessed with Fluoro-Ruby retrograde labeling and Nissl staining.

Results: This study showed that GDNF allowed an increase in the number and the maturation of nerve fibers, as well as the number of retrogradely labeled neurons in delayed anastomoses. On the contrary, after immediate repair, the regenerated nerves in the presence of GDNF showed inferior results compared to the other groups.

Conclusions: GDNF is a potent neurotrophic factor to improve facial nerve regeneration in grafts performed several months after the nerve lesion.

From the Department of Otorhinolaryngology (F.M.B., P.P.); and Nerve-Muscle Unit, Neurology Service (т.к.), Department of Surgical Research (A.D.Z.), CHU Vaudois and University of Lausanne, Lausanne, Switzerland.

Editor's Note: This Manuscript was accepted for publication December 4, 2008.

This study has been supported by the Novartis Foundation.

Send correspondence to Florian M. Barras, Department of Otorhinolaryngology, BH-12, 1011 Lausanne-CHUV, Switzerland. E-mail: florian.barras@chuv.ch

DOI: 10.1002/lary.20169
However, GDNF should not be used for immediate repair, as it possibly inhibits the nerve regeneration.

Key Words: Glial cell line-derived neurotrophic factor, nerve grafting, Schwann cells, nerve guidance channel.

Laryngoscope, 119:846-855, 2009

\section{INTRODUCTION}

Facial nerve paralysis is a devastating clinical condition leading to severe aesthetic and functional alterations. When a surgical repair is indicated, the appropriate technique is chosen according to the nature and location of the lesion, the age of the patient, and the delay between the nerve lesion and its repair. Although direct end-to-end, tension-free coaptation is the most effective procedure, a nerve gap often requires an autologous nerve graft harvested on the sural or auriculotemporal nerves. ${ }^{1}$ Nevertheless, poor functional results are observed for mandibular or frontal branches grafting, ${ }^{2-4}$ in the case of prolonged delay between the lesion and the repair and by aged patients. ${ }^{5,6}$ The expected reasons could be the limited number of regenerated fibers or inefficacy of Schwann cells to support regeneration in such clinical situations. Hypoglossal-facial anastomosis is another effective technique when the proximal facial nerve stump is not available for grafting (after pontocerebellar angle tumor removal or centrally occurring lesions). ${ }^{7}$ Nevertheless, results can be compromised also in older patients or in case of prolonged delay between the lesion and the repair. Cross-facial nerve grafting has been described as an alternative to hypoglossal-facial anastomosis and consists in connecting peripheral fibers of the facial nerve selected on the healthy side to the lesioned side via an autologous nerve graft. ${ }^{8}$ The results, however, have been disappointing due to the insufficient number and the immaturity of the regenerating nerve fibers, associated with the small amount of fibers available in the donor site and the length of the nerve gap. ${ }^{9}$ For these reasons, cross-facial nerve grafting is nowadays only used in combination with other techniques such as hypoglossal-facial 
anastomosis (babysitter procedure), ${ }^{10,11}$ or free muscle transfer (m. gracilis, $\mathrm{m}$. pectoralis minor, etc.). ${ }^{12}$

All surgical techniques described above have largely contributed to reanimate a paralyzed face, yet with inconsistent results related to the anatomical and individual variability. In order to obtain more efficient nerve regeneration, the next step is to target the nerve at a molecular level. Indeed, neurotrophic factors delivered through nerve guidance channels (NGCs) have been demonstrated to be valuable tools for this purpose. Neurotrophic factors are naturally occurring proteins implicated in nervous system development, survival, and repair. ${ }^{13}$ Among the different classes of neurotrophic factors, the glial cell line-derived neurotrophic factor (GDNF), has been demonstrated to have potent regenerating effects on peripheral nerves. ${ }^{14-16}$ It increases the number of regenerating nerve fibers, improves their maturity and protects the neural cell bodies. ${ }^{14,17,18}$ This has been demonstrated in the sciatic as well as in the facial nerve, with GDNF delivered locally on the site of regeneration via nerve guidance channels during several weeks. ${ }^{19,20}$ The aim of this study was to evaluate the effect of GDNF locally delivered via a nerve guidance channel on autologous nerve graft after immediate and delayed repair of the mandibular branch of the rat facial nerve.

\section{MATERIALS AND METHODS}

\section{GDNF-Releasing Nerve Guidance Channels}

NGCs were made of a nonresorbable ethylene vinyl acetate (EVA) polymer tube, into the wall of which an EVA rod releasing GDNF was incorporated as described previously. ${ }^{21,22}$ The recombinant neurotrophic factor GDNF was provided by Amgen Inc. (Thousands Oak, CA). After implantation, the neurotrophic factor was released progressively over several weeks. In vitro GDNF release was evaluated using an ELISA assay as described previously. ${ }^{19}$ The channels used in the present study were taken from the same batches as those used earlier. ${ }^{19}$

\section{Groups of Animals}

Two main groups of 20 adult male Wistar rats each were constituted. In the first group (immediate repair), the nerve grafting procedure was performed immediately after the nerve lesion. In the second group (delayed repair), the nerve repair was achieved 7 months after the facial nerve lesion. Each group was divided in three different subgroups: 1) autologous nerve graft of $15 \mathrm{~mm}$ without channel, nor GDNF, $\mathrm{n}=4$ (graft alone); 2) autologous nerve graft of $12 \mathrm{~mm}$ with a regular suture on the proximal stump and a $5 \mathrm{~mm}$ channel without GDNF between the distal nerve stump and the graft, separated by an internal gap of $3 \mathrm{~mm}, \mathrm{n}=6$ (graft, channel without GDNF); and 3) same as subgroup 2, but with a channel liberating GDNF, $\mathrm{n}=10$ (graft, channel GDNF).

\section{Surgical Procedures}

Adult male Wistar rats of approximately $300 \mathrm{~g}$ were anesthetized with isoflurane delivered with a Minerve anesthetic apparatus. All animal protocols were approved by the local veterinary commission in Lausanne, Switzerland, and carried out in accordance with the European Community Council directive $86 / 609 / \mathrm{ECC}$ for the care and use of laboratory animals. Rats
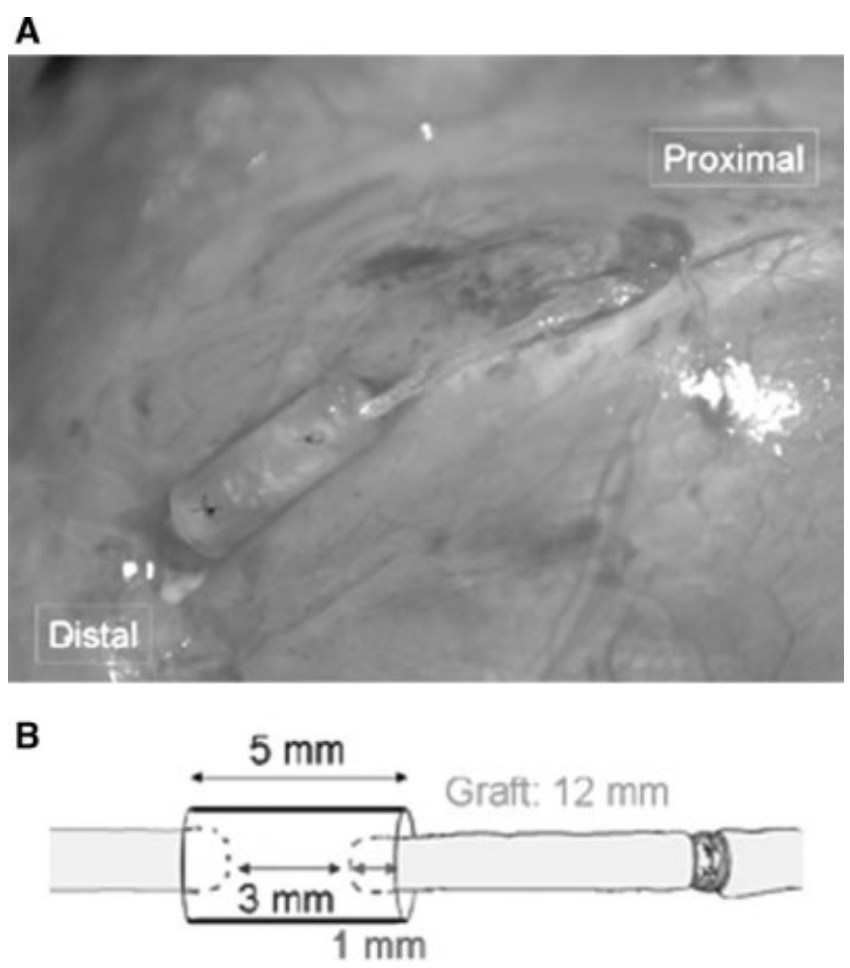

Fig. 1. (A) Photograph showing a 0.5-cm-long channel implanted on the distal suture of an autologous nerve graft on the mandibular branch of the facial nerve. (B) Scheme of the system with a $5 \mathrm{~mm}$ channel on the distal suture. Each nerve stump is inserted $1 \mathrm{~mm}$ into the channel, creating a 3-mm internal gap.

were shaved in the facial area up to the shoulder, in the region of sural nerve harvesting, on the dorsal face of the left leg. Local disinfection was performed with a 25\% Betadine $/ 0.9 \%$ $\mathrm{NaCl}$ solution. All surgical procedures were performed using a surgical microscope (Zeiss, Oberkochen,Germany). For sural nerve harvesting, the left leg was opened on the posterior side with a longitudinal and medial incision. The sural nerve was isolated, avoiding its compression and preserving the local vasculature. It was cut proximally and distally in order to obtain a segment of at least $18 \mathrm{~mm}$ and prepared to obtain a unique fascicule. For facial nerve repair, the skin was opened with a preauricular and cervical incision on the left side. The mandibular branch of the facial nerve was exposed close to the stylomastoid foramen and cut distally to the bifurcation in order to preserve the zygomatic branch. A $10-\mathrm{mm}$ segment of the mandibular branch was removed before it gave rise to the marginal branches. This $10-\mathrm{mm}$ gap was then replaced by a $15-\mathrm{mm}$ autologous nerve graft or a $12-\mathrm{mm}$ nerve graft with a 5 -mm channel on the distal suture (internal gap of $3 \mathrm{~mm}$ ) (Fig. 1A, B). The whole system measured $15 \mathrm{~mm}$ in all animals, with or without channel on the distal nerve stump. Each side of the nerve was inserted $1 \mathrm{~mm}$ into the channel and fixed with one 10-0 trans-epineurial Ethilon suture (Ethicon/Johnson \& Jonhson, Spreitenbach, Switzerland), leaving an internal nerve gap of $3 \mathrm{~mm}$ (Fig. 1B). The proximal suture was fixed with three 11.0 nylon sutures. The channel was then fixed locally into the masseter muscle with 3x 6-0 Prolene sutures (Ethicon/Johnson \& Jonhson, Spreitenbach, Switzerland) to reduce movements due to scratching. Skin was closed with a nylon 5-0 Dermalon (Sherwood-Davis \& Geck, Adliswil, Switzerland). Animals were housed during 6 weeks in a controlled environment with a 12 hour on-off light cycle and water and food ad libitum. 


\begin{tabular}{cl}
\hline & TABLE I. \\
& Quality of Contraction of Facial Muscles. \\
\hline Score & \multicolumn{1}{c}{ Observations } \\
\hline 0 & No function, asymmetry at rest \\
1 & Symmetry at rest, but no voluntary movement \\
2 & Symmetry at rest, weak voluntary contractions \\
3 & Symmetry at rest, moderate voluntary contractions \\
4 & $\begin{array}{c}\text { Symmetry at rest, good voluntary contraction, but } \\
\text { persisting asymmetry in comparison with the } \\
\text { unlesioned side } \\
\text { Normal function, perfect symmetry compared to the } \\
\text { unlesioned side }\end{array}$ \\
\hline \hline
\end{tabular}

All the animals were observed at 3 and 6 weeks. A score (0-6) was attributed to each animal.

The animals included into the group of delayed repair were first operated on the facial area. The facial nerve was sectioned and a segment of $5 \mathrm{~mm}$ was removed. The two stumps were ligatured in order to prevent a spontaneous regeneration. After 7 months, the rats were reopened and the facial nerve stumps were located. The two nerve stumps were recut in order to remove the fibrous tissue and the same procedure was performed as in the group with an immediate repair.

\section{Functional Evaluation}

Functional observation. The animals were examined to evaluate the rapidity of nerve regeneration. The quality of contraction of the facial muscles (whiskerpads) was assessed and a score was attributed to each animal, as described in the Table I. The observations were performed at 3 and at 6 weeks after nerve repair by two blinded observers.

Nerve conduction study. Nerve conduction (NC) studies were performed 6 weeks after nerve repair (just after the second clinical observation). The rats were maintained at a constant temperature of $37^{\circ} \mathrm{C}$ on a warming plate. In order to prevent recording muscle contractions from the lateral whiskerpad, the buccal branch of the facial nerve was sectioned just prior to the study. Ground, recording, and stimulating monopolar electrodes (Carbamed, Zurich, Switzerland) were inserted subcutaneously. The active recording electrode was inserted at the level of the chin, whereas the reference electrode was placed in the interocular area. A ground electrode was inserted in the front of the head. The stimulating electrodes were placed distally to the mandibular branch of the facial nerve. The supramaximal compound muscle action potential (CMAP) was recorded by progressive incremental stimulations and the negative area, expressed in $\mathrm{msmV}$, was used for statistical analysis.

\section{Retrograde Labeling}

Four days before sacrifice, and immediately after NC studies, the animals were anesthetized as described under surgical procedures, the operative site was reopened, and the facial nerve was exposed and cut $8 \mathrm{~mm}$ distally to the channel, just before it ramifies. Five microliters of a $5 \%$ Fluoro-ruby solution (dextran, tetramethylrhodamine) (Molecular Probes, Life Technologies Corporation, Carlsbad, CA) in $0.9 \% \mathrm{NaCl}$ were delivered via a small cup fixed to the nerve with Histoacryl glue (Braun, Sempach, Switzerland).

Channel and regenerated nerve retrieval. Six weeks after surgery and 4 days after Fluoro-ruby delivery, the rats were deeply anesthetized with a lethal intraperitoneal injection of pentobarbital and perfused transcardially with $200 \mathrm{~mL}$ hep- arinized PBS followed by $200 \mathrm{~mL}$ of $4 \%$ paraformaldehyde in PBS pH 7.4. The operative site was reopened and the NGCs and regenerated nerves (graft + channel $+5 \mathrm{~mm}$ beyond the graft) were removed and postfixed for 15 days in PBS pH 7.4 containing $4 \%$ paraformaldehyde and $1 \%$ glutaraldehyde. The brainstems were harvested, postfixed overnight in $4 \%$ paraformaldehyde in PBS pH 7.4 and submerged in a 25\% sucrose solution for 72 hours prior to cryosection.

\section{Morphometric Analysis}

The regenerated nerves were processed as described in a former study. ${ }^{19}$ They were cut in the graft (in the middle of the tube or $3 \mathrm{~mm}$ before the distal suture in the groups without channel), and $4 \mathrm{~mm}$ distally to the channel. Myelinated axon counts and morphometric analysis were performed under microscopy at a magnification of $1,000 \times$ (objective $100 \times$ and ocular $10 \times$ ). The maturation of the regenerated nerve fibers was assessed by measuring the myelin thickness. The measurements were performed randomly using an image analysis system (analysis soft imaging system).

To assess the number of retrogradely labeled facial motoneurons, the brainstems were harvested, frozen on dry ice, embedded in Tissue-Tek OCT compound (Miles, Streuli Rudolph AG, Switzerland), and $20-\mu \mathrm{m}$ sections were cut using a cryostat Cryocut 1800 (Leica, Wetzlar, Germany). Retrogradely labeled neurons were counted using an Olympus PX 40 fluorescence microscope (Olympus, Center Valley, PA) with a wild-band UV filter $(200 \times$ magnification). The total number of labeled motoneurons in the facial nucleus was obtained by multiplying by 5 the number of neurons counted on every fifth section.

Neuronal survival. The total number of motoneurons present in the facial nucleus was evaluated in Nissl-stained sections. Only well-marked motoneurons with a clearly visible nucleus and located in the facial nucleus area were counted in every fifth section. The total number of counted motoneurons was multiplied by 5 to obtain the total number of motoneurons in the facial nucleus. The results were expressed as a percent of motoneuron loss in the lesioned side compared to the unlesioned side.

\section{Statistical Analysis}

All the data were analyzed for statistical significance using a nonparametric analysis of variance (ANOVA) with a Kruskall-Wallis test.

\section{RESULTS}

The results of the in vitro releases and bioactivity assays performed with the nerve guidance channels containing GDNF were reported in details in a previous paper. $^{19}$

Approximately $35 \%$ of all channels were found to be disconnected at sacrifice, probably due to scratching (21 analyzable animals out of 32 implanted animals). The animals with disconnected channels were not taken into account for further analysis. Thus, only $4 / 6$ animals in the group, with channel without GDNF, and 8/10 animals in the group with channel with GDNF, could be analyzed in the immediate repair group, whereas 5/6 animals in the group, with channel without GDNF, and 6/10 animals in the group with channel with GDNF, could be analyzed in the delayed repair group. 
TABLE II.

Facial Contractions: Immediate Repair Group.

\begin{tabular}{lcc}
\hline & Score at 3 wk & Score at 6 wk \\
\hline Graft without channel & $2.5 \pm 0.3$ & $3.3 \pm 0.3$ \\
Graft and channel without GDNF & 3.0 & $3.8 \pm 0.4$ \\
Graft and channel with GDNF & $3.0 \pm 0.3$ & $3.7 \pm 0.4$ \\
\hline \hline
\end{tabular}

GDNF = glial cell line-derived neurotrophic factor.

No statistical difference was found between the different subgroups at 3 and 6 weeks.

\section{Immediate Repair}

As mentioned above, the subgroup with channel without GDNF contained 4 analyzable animals $(\mathrm{n}=4)$, and the GDNF subgroup contained 6 animals $(n=6)$. All animals of the normal graft subgroup were analyzable $(\mathrm{n}=4)$.

The observation of facial contractions at 3 weeks gave similar scores in the different groups (no statistical difference): a score of $2.5 \pm 0.3$ points in the subgroup with a normal graft, $3.0 \pm 0$ points in the subgroup with a distal channel delivering no neurotrophic factor, and $3.0 \pm 0.3$ points in the subgroup with a graft with GDNF releasing channels (Table II). Similarly, there was no statistical difference between the different subgroups at 6 weeks. In the GDNF subgroup a score of 3.7 \pm 0.4 was calculated, the normal graft subgroup gave a mean score of $3.3 \pm 0.3$, and the subgroup with channel without neurotrophic factor had a score of $3.8 \pm 0.4$ (Table II).

Considering the NC parameters, there was no statistical difference between the different groups. The mean CMAP area was $2.4 \pm 0.46 \mathrm{mVms}$ in the graft alone subgroup, $1.69 \pm 0.58 \mathrm{mVms}$ in the subgroup with channel without neurotrophic factor, $3.05 \pm 0.84 \mathrm{mVms}$ in the subgroup with channel delivering GDNF, and 3.79 $\pm 0.12 \mathrm{mVms}$ when recorded in the unlesioned side (Fig. 2A).

The pictures obtained in the group with immediate repair are shown in the Figure 3B-D (in the graft) and Figure 4A-C (distal to the graft). Myelinated axons were found in each group. Nerves regenerated in normal conditions of graft, without channel, showed 1,809 \pm 106 myelinated axons in the distal part of the graft, and $1,905 \pm 338$ myelinated axons distally to the graft. Grafts with a control channel allowed to obtain 2,042 \pm 299 myelinated axons in the middle of the tube, and $2,008 \pm 522$ myelinated axons distal to the graft. In the grafts regenerated in the presence of GDNF, 3,268 \pm 791 myelinated axons were counted in the middle of the channel and 1,077 \pm 248 distally to the graft. There was no statistical difference between the GDNF subgroup and other subgroups. As a comparison, a naive nerve contains $1,404 \pm 36$ axons. Similarly, there was no statistical difference between the different repaired groups when measuring the myelin thickness.

The pictures of retrogradely labeled motoneurons of the facial nucleus are shown in Figure 5A-D. The retrograde labeling performed on the control side showed a total number of $2,207 \pm 95$ labeled neurons. In this group, the number of retrogradely labeled neurons is significantly lower in the GDNF subgroup $(1,059 \pm 73)$ than in the graft alone subgroup $(1,739 \pm 86)(P<.01)$ and lower than in the subgroup with channel without GDNF $(1,489 \pm 114)(P<.03)$. Similarly, neuronal loss on the lesioned side, compared to the unlesioned side, was significantly higher in the GDNF subgroup (16.4 $\pm 2 \%$ of difference) than in the other groups $(10.9 \pm 1.7 \%$ in the subgroup channel without GDNF, $P<.001$; and $1.1 \pm$ $0.6 \%$ in the graft alone subgroup, $P<.001$ ) (Fig. 6A).

\section{DELAYED REPAIR}

In the group of delayed repair, all the repaired animals of the normal graft subgroup were analyzable $(\mathrm{n}=$ 4), although one rat in the subgroup channel without GDNF and five rats of the GDNF subgroup had to be discarded because of channel disconnection. Thus, the subgroup channel without GDNF contained five analyzable animals $(\mathrm{n}=5)$, and the GDNF subgroup contained 5 animals also $(\mathrm{n}=5)$.

The observation of facial contractions at 3 weeks gave a score of $1 \pm 0$ point in the subgroup with a normal graft, $1.2 \pm 0.2$ point in the subgroup with a distal channel delivering no neurotrophic factor, and $2.3 \pm 0.3$ points in the subgroup with a graft with GDNF
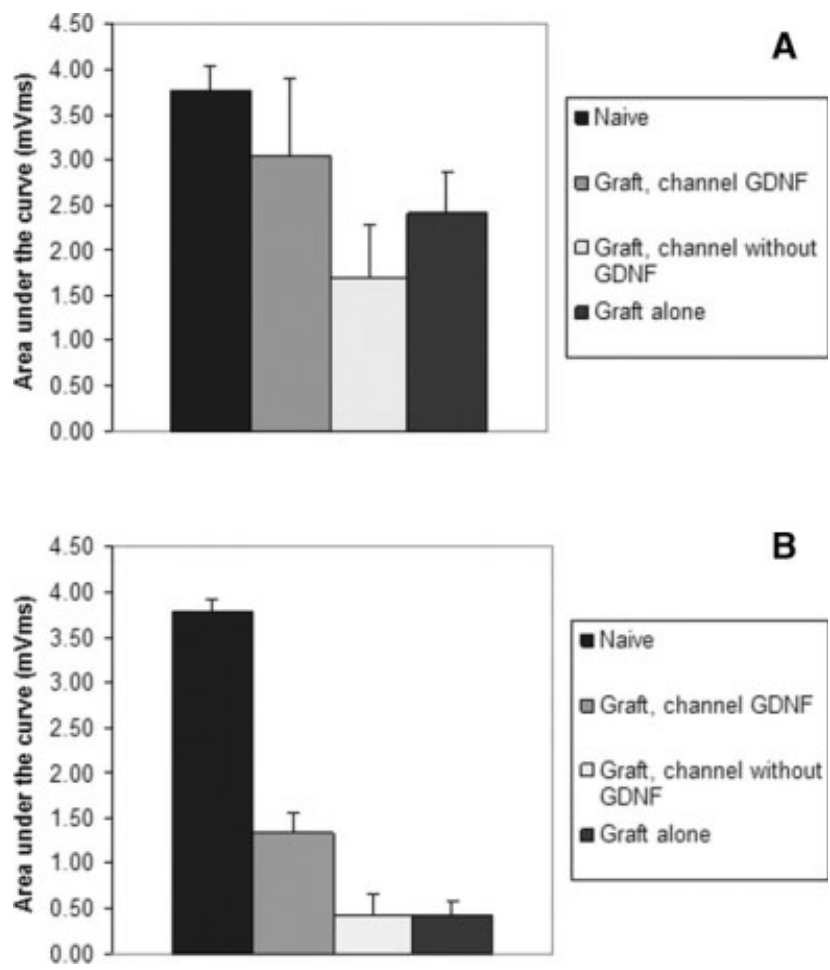

Fig. 2. Electromyogram measured at 6 months, just before nerve transection for retrograde labelling. (A) Immediate repair group; no statistical difference was found between the different repaired groups. (B) Delayed repair group; the surface measured under the curve was significantly larger in the GDNF subgroup compared o the other repaired groups $(P<.02$ when comparing the GDNF subgroup with the normal graft subgroup, and $P<.03$ when comparing the GDNF subgroup with the subgroup channel without GDNF). 

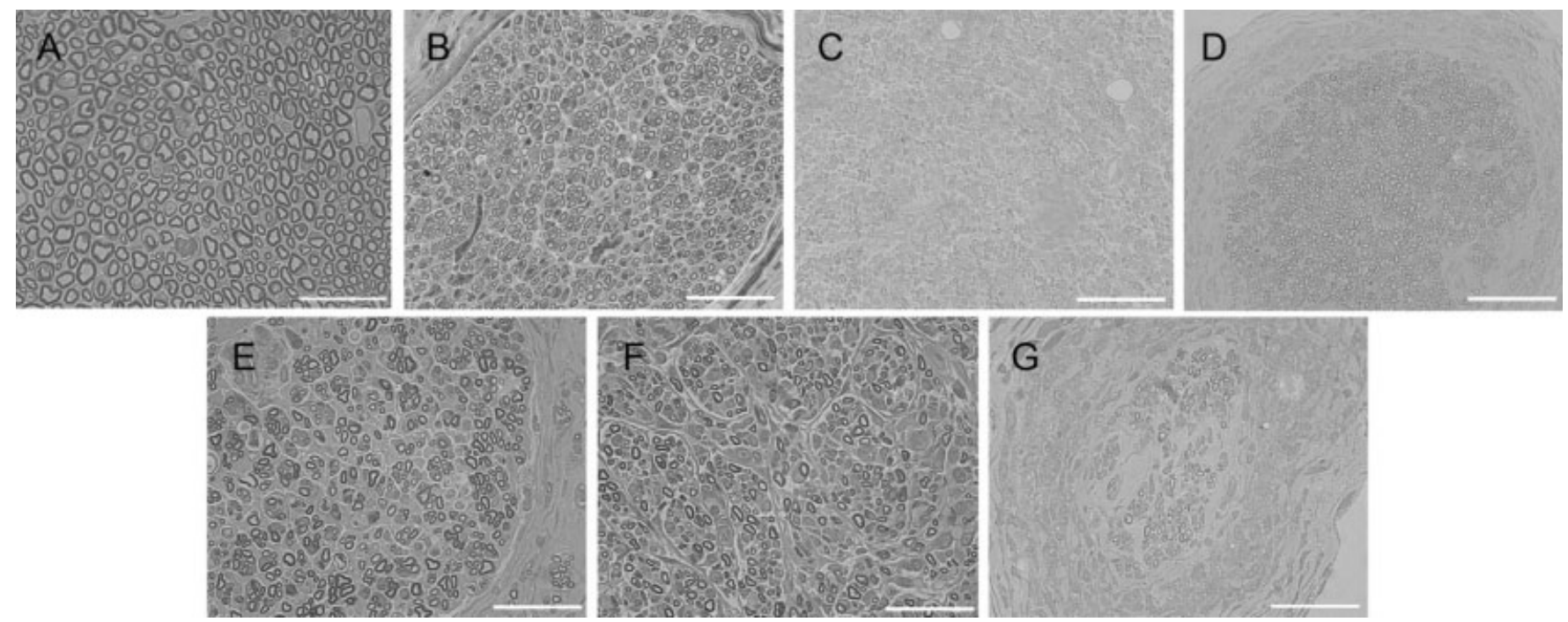

Fig. 3. Photomicrographs of facial nerves regenerated in the distal part of the graft. (A) Represents a normal naive mandibular branch of the facial nerve. (B-D) Immediate repair. (B) Graft alone, section $3 \mathrm{~mm}$ from the distal suture; (C) Graft with channel delivering GDNF; (D) Channel without GDNF. Original magnification, 1,000x; scale bars $=20 \mu \mathrm{m}$. The total number of myelinated fibers has been evaluated for each group. Statistical significance was evaluated using the nonparametric Kruskall-Wallis test. No statistical significance has been found between the different repaired groups. (E-G) Delayed repair. (E) Normal graft; (F) Graft with channel delivering GDNF; (G) Channel without GDNF. Original magnification, 1,000×; scale bars $=20 \mu \mathrm{m}$. The total number of myelinated fibers has been evaluated for each group. Statistical significance was evaluated using the nonparametric Kruskall-Wallis test. The subgroup with GDNF-delivering channels showed a significantly larger number of myelinated nerve fibers than the normal graft subgroup and the subgroup with channel delivering no GDNF $(P<.03$ and $P<.01$, respectively).

releasing channels, respectively (Table III). The difference between the GDNF subgroup and the two other subgroups was significant $(P<.01$, GDNF compared with normal graft; and $P<.02$, GDNF compared with channel without GDNF). At 6 weeks, the differences between the different subgroups were not as pronounced as at 3 weeks, but a tendency for a better function in the GDNF subgroup was observed, with a score of $4.3 \pm$
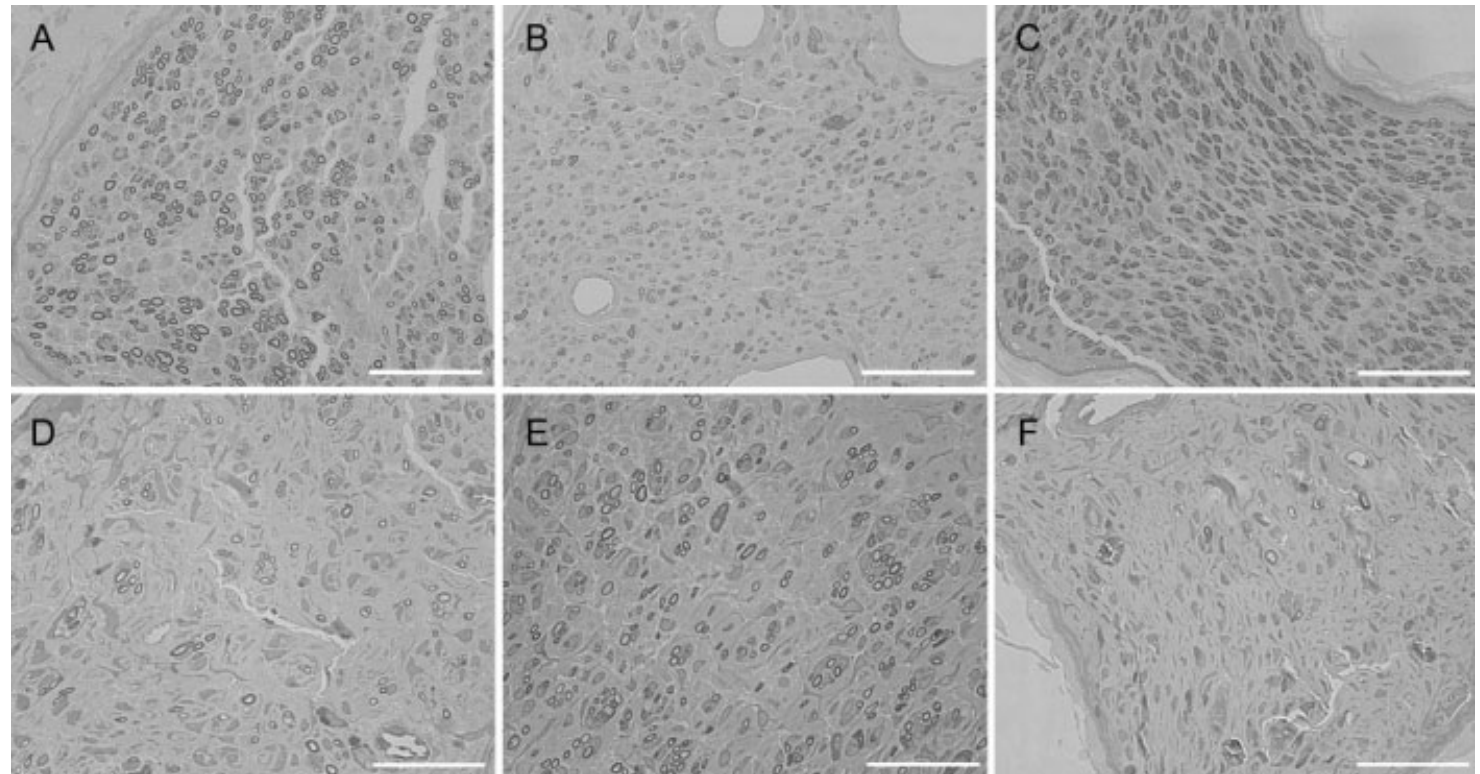

Fig. 4. Photomicrographs of facial nerve regenerated $4 \mathrm{~mm}$ distally to the graft. (A-C) Immediate repair. (A) Graft alone; (B) Graft with channel delivering GDNF; (C) Channel without GDNF. The total number of myelinated fibers has been evaluated for each group. Statistical significance was evaluated using the nonparametric Kruskall-Wallis test. No statistical significance has been found between the different repaired groups. (D-F) Delayed repair. (D) Graft alone; (E) Graft with channel delivering GDNF; (F) Channel without GDNF. Original magnification, 1,000x; scale bars $=20 \mu \mathrm{m}$. The total number of myelinated fibers has been evaluated for each group. Statistical significance was evaluated using the nonparametric Kruskall-Wallis test. The subgroup with GDNF-delivering channels showed a significantly larger number of myelinated nerve fibers than the normal graft subgroup and the subgroup with channel delivering no GDNF $(P<.03$ and $P<.02$, respectively). 

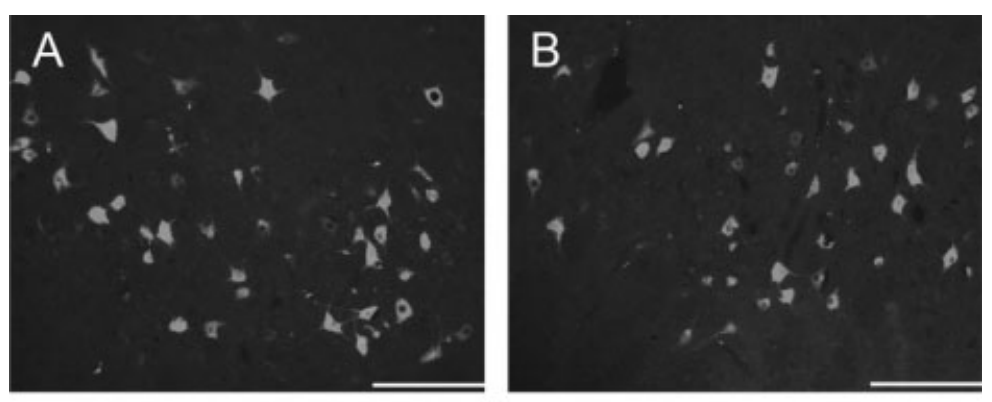

Fig. 5. Retrograde tracing of facial motoneurons. Micrographs showing Fluororuby-labeled motoneurons in the facial nucleus. (A-D) Immediate repair group; (A) Retrograde tracing performed in an unlesioned rat; (B) Graft alone; (C) Graft, channel GDNF; and (D) Graft, channel without GDNF. Original magnification, $100 \times$; scale bars $=200 \mu \mathrm{m}$. The quantification of Fluororuby retrogradely labeled motoneurons into the facial nucleus showed a significantly larger number of labeled neurons in the subgroup with normal graft, as well as the subgroup of graft, channel without GDNF compared to the subgroup with graft and channel delivering GDNF $(P<.01$, and $P<.03$, respectively). (E-H) Delayed repair group; (E) Retrograde tracing performed in an unlesioned rat; $(F)$ Graft alone; $(G)$ Graft, channel GDNF; and $(\mathrm{H})$ Graft, channel without GDNF. Original magnification, 100 $\times$; scale bars $=200 \mu \mathrm{m}$. The number of retrogradely labeled motoneurons was significantly larger in the group of graft with GDNF compared to normal graft $(P<.03)$ and compared to the group graft and channel without GDNF $(P<.01)$.
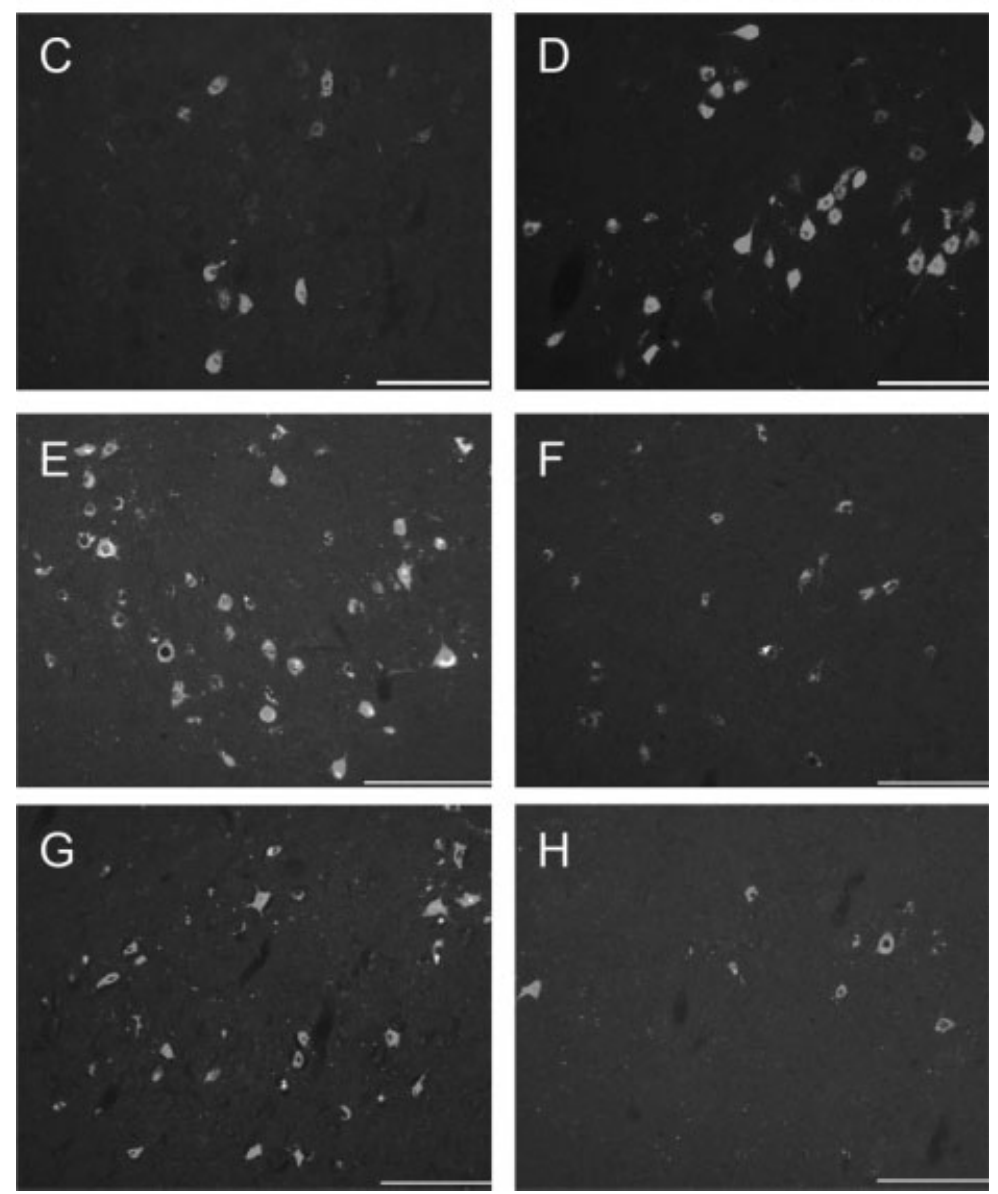

0.3 , whereas the graft alone subgroup gave a mean score of $3.3 \pm 0.3$, and the subgroup channel without GDNF had a score of $3 \pm 0.3$ (Table III).

Considering the NC parameters, the CMAP area was significantly larger in the GDNF subgroup (1.34 \pm $0.21 \mathrm{mVms}$ ), whereas it measured $0.42 \pm 0.15 \mathrm{mVms}$ in the group with a graft alone $(P<.02), 0.43 \pm 0.24$ $\mathrm{mVms}$ in the subgroup with channel without GDNF $(P$ $<.03$ ), and $3.79 \pm 0.12 \mathrm{mVms}$ in the unlesioned side (Fig. 2B).

The pictures obtained in the group with delayed repair are shown in the Figure $3 \mathrm{E}-\mathrm{G}$ (distal part of the graft) and Figure 4D-F (distally to the graft). Myelinated axons were found in each subgroup. Nerves regenerated in normal conditions of graft, without channel showed 1,185 \pm 101 myelinated axons in the distal part of the graft, and $655 \pm 130$ myelinated axons dis- tally to the graft. Grafts with a channel without GDNF resulted in $335 \pm 113$ myelinated axons in the distal part of the graft (middle of the channel), and $214 \pm 114$ myelinated axons distally to the graft. In the grafts regenerated in presence of a GDNF delivering channel, the number of myelinated axons was larger at the two sites of analysis: 3,204 \pm 747 myelinated axons in the distal part of the graft (middle of the channel), and $1,608 \pm 363$ myelinated axons distally to the graft. As a comparison, a naive nerve contains 1,286 \pm 43 axons. When analyzing the different subgroups for significance, the GDNF subgroup showed a significantly larger number of myelinated fibers into the graft than the normal graft subgroup $(P<.03)$, as well as the channel without GDNF subgroup $(P<.01)$. Similarly, the GDNF subgroup showed a significantly larger number of myelinated fibers distally to the graft than the normal 
A

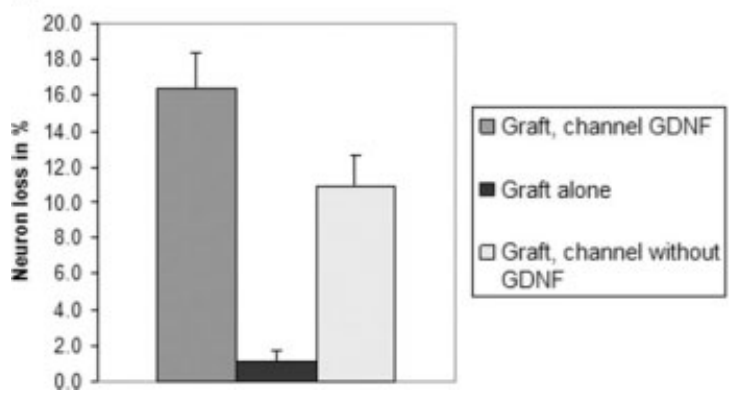

B

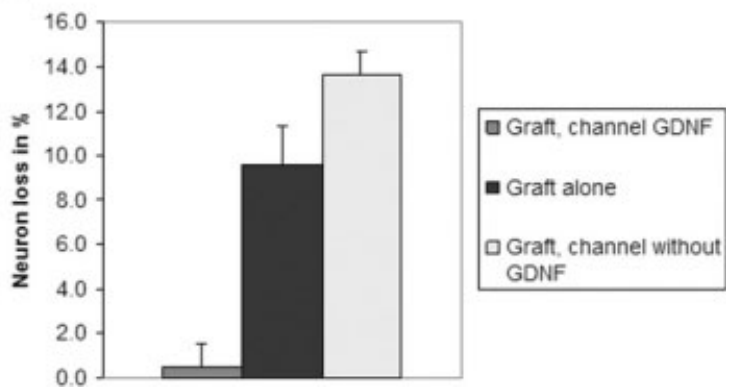

Fig. 6. Motoneuron loss on the lesioned side was expressed as a percentage of the unlesioned side. (A) Immediate repair group; the neuronal loss was significantly larger in the channel GDNF group than in the two other groups $(P<.001$ for both); (B) Delayed repair group; the neuronal loss was significantly lessened in the channel GDNF group than in the subgroup with graft alone and the subgroup channel delivering no GDNF $(P<.009$ and $P<.001$, respectively).

graft subgroups and the channel without GDNF subgroup $(P<.03$ and $P<.02$, respectively).

The myelin thickness was assessed as a measure of maturation of the regenerated fibers. This measure was performed in the distal part of the graft and distally to the graft. In all cases, the myelin thickness of the regenerated axons was smaller than that of a naive nerve $(0.98 \pm 0.01 \mu \mathrm{m})$ (Fig. 7). In the distal part of the graft, myelin thickness was larger in the graft alone subgroup $(0.41 \pm 0.01 \mu \mathrm{m})$ than in the two other groups $(0.28 \pm$ 0.01 and $0.35 \pm 0.02 \mu \mathrm{m}$ in the subgroup with channel without neurotrophic factor and GDNF channel, $P<.05$, respectively). However, distally to the graft, the myelin thickness became significantly larger in the presence of GDNF $(0.34 \pm 0.01 \mu \mathrm{m})$ than in the groups without neurotrophic factor $(0.3 \pm 0.01, P<.03$; and $0.27 \pm 0.01, P$ $<.02 \mu \mathrm{m}$ in the graft alone and channel without GDNF, respectively).

Micrographs of retrogradely labeled motoneurons in the facial nucleus are shown in Figure 5E-H. The counts of retrogradely labeled motoneurons in the facial nucleus revealed a total number of $1,516 \pm 98$ motoneurons in the unlesioned animals. In the presence of GDNF, 1,051 \pm 200 retrogradely labeled neurons were counted, whereas only $410 \pm 142$ and $257 \pm 101$ labeled neurons were present in the graft alone subgroup and in the channel without GDNF subgroup, respectively.

The neuronal loss on the lesioned side compared to the unlesioned side showed very little difference in the

TABLE III.

Facial Contractions: Delayed Repair Group.

\begin{tabular}{lcc}
\hline & Score at 3 wk & Score at 6 wk \\
\hline Graft without channel & 1 & $3.3 \pm 0.3$ \\
Graft and channel without GDNF & $1.2 \pm 0.2$ & $3 \pm 0.3$ \\
Graft and channel with GDNF & $2.3 \pm 0.3$ & $4.3 \pm 0.3$ \\
\hline \hline
\end{tabular}

GDNF $=$ glial cell line-derived neurotrophic factor.

At 3 weeks, the score obtained in the GDNF subgroup was significantly better than in the normal graft subgroup and in the graft with channel without GDNF subgroup ( $P<.01$ and $P<.02$, respectively). No statistical difference was found between the different subgroups at 6 weeks. subgroup with GDNF $(0.5 \pm 1.1 \%)$, whereas the difference was much larger in the other groups $(13.6 \pm 1.0 \%$ in the channel without GDNF subgroup, and $9.6 \pm 1.7 \%$ in the graft alone subgroup) (Fig. 6B). These differences were statistically significant, $P<.009$, when comparing the subgroup with the subgroup graft alone, and $P<$ .001 , when comparing the subgroup channel delivering GDNF with the subgroup channel without GDNF.

A

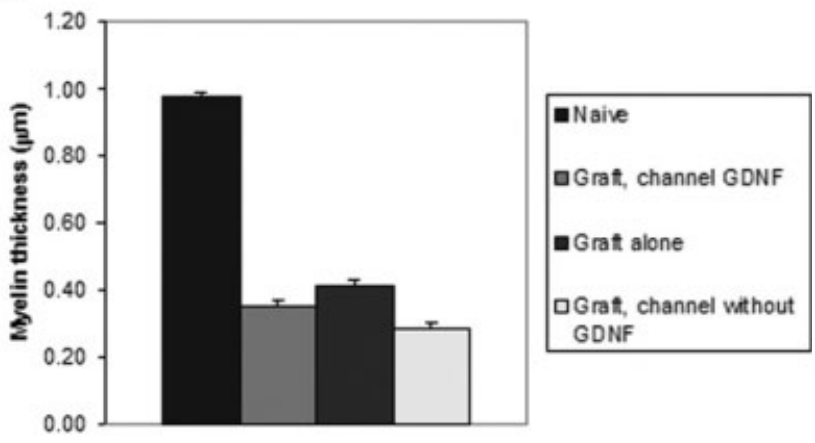

B

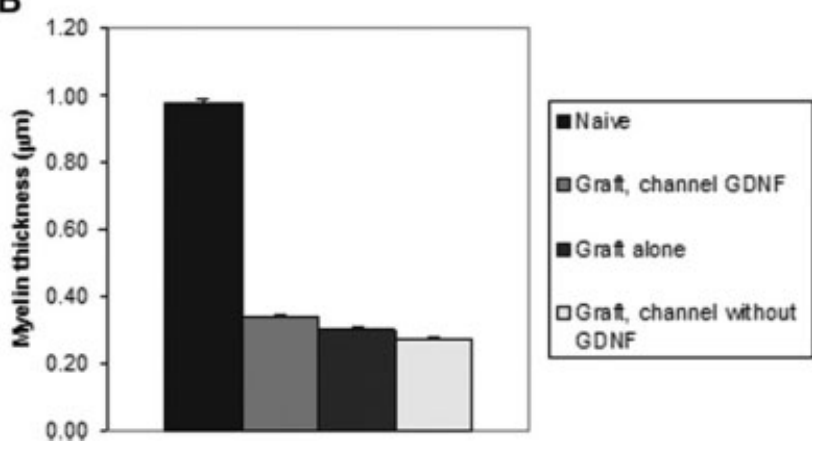

Fig. 7. Quantification of myelin thickness as a measure of maturity of regenerated nerves in the delayed repair group. (A) In the distal part of the graft; the myelin thickness was significantly larger in the normal graft group compared to graft with channel GDNF $(P$ $<.05$ ); (B) $5 \mathrm{~mm}$ distally to the graft; the myelin thickness was significantly larger in the subgroup of graft with GDNF compared with normal graft subgroup $(P<.03)$ as well as the channel without GDNF subgroup $(P<.02)$. 


\section{DISCUSSION}

Recovery after facial nerve injury is often poor despite the various grafting techniques applied in the clinic. This is the case particularly in lesions of the mandibular or frontal branches, when cross-facial nerve grafts have to be performed in aged patients and when the delay between the lesion and its repair is prolonged..$^{3,5,23,26}$ So far, facial nerve regeneration with local application of nerve growth factor or brain derived neurotrophic factor, two neurotrophic factors of the neurotrophin family, had revealed only limited improvement. ${ }^{27,28}$ Our previous study demonstrated that GDNF is a potent neurotrophic factor, which improves facial nerve regeneration in the peripheral nervous system, and promotes survival in the central nervous system. ${ }^{19}$ However, this study was not designed for clinical purposes, but to identify the most potent neurotrophic factor for facial nerve regeneration. The present study focused on a more clinical model, consisting in improving facial nerve regeneration with the local application of GDNF on the distal sutures of autologous nerve grafts performed on the mandibular branch of the facial nerve. The distal placement of the channel was determined according to the literature. ${ }^{29}$ Indeed, in cases of silicone channels delivering no neurotrophic factors, it has been determined that the distal placement of a channel had better effects than its proximal or both-sites placements. $^{29}$ Different studies allowed to fix the ideal internal gap to $3-4 \mathrm{~mm} .^{30-34}$ Similarly, it has already been determined that an entubulated end-to-end coaptation has no effect on nerve regeneration. ${ }^{35}$

Continuous release of nanogram quantities of GDNF on the distal suture of autologous nerve grafts over a period of 6 weeks gave opposite results in the two groups of the study, i.e., immediate or 7-month delayed repair. In the immediate repair group, no, and even a tendency of worse results, were obtained with GDNF. On the contrary, a delayed repair allowed for much better results in the presence of GDNF.

In the immediate repair group, the subgroup with GDNF showed significantly fewer regenerated nerve fibres in the distal nerve stump (distally to the channel), no improved maturation, a significantly lower number of retrogradely labeled motoneurons in the facial nucleus, as well as a larger percent of motoneuron loss on the lesioned side. It has previously been shown that a peripheral nerve crush leads to a rapid up-regulation of the GDNF mRNA in the distal nerve stump, an increase of GDNFR-alpha receptor in the distal nerve stump, and an up-regulation of the Ret mRNA in the motor neurons. ${ }^{18,36,37}$ A facial nerve lesion induces an upregulation of the mRNAs of the GDNFR-alpha-, c-ret-, and vesicle-associated membrane proteins receptors in the facial motor neurons. ${ }^{17,38}$ However, unpublished results obtained in our previous studies allowed us to establish that large amounts of GDNF delivered at the lesion site had deleterious effects, with even an inhibition of nerve regeneration in cases of overdose. The lessened regeneration obtained in cases of immediate repair with the addition of exogenous GDNF could be due to a too large amount of neurotrophic factor GDNF. Indeed, exogenous GDNF added to the in vivo delivered GDNF could produce an excessive dose of neurotrophic factor, thus leading to deleterious effects on nerve regeneration.

On the contrary, when the delay between the lesion and its repair is prolonged, the application of GDNF to a chronically denervated nerve gave significantly better results than repairs without neurotrophic factor (graftalone subgroup and graft with a channel without GDNF subgroup). In this situation, functional results and the CMAP areas measured at EMG were significantly better for the GDNF subgroup. The number of regenerated myelinated nerve fibers and the maturity of the regenerated fibers were also significantly better in the GDNF subgroup. Similarly, the central patterns were significantly better in the nerve regenerated in the presence of GDNF (significantly larger number of retrogradely labeled motoneurons and significantly less neuronal loss when comparing the lesioned versus the unlesioned facial nuclei). In this situation, the exogenous supply of GDNF allowed significantly improved facial nerve regeneration. It has been demonstrated that in cases of prolonged denervation, the Schwann cells of the distal nerve stump are no more responsive to a front of regenerating nerve fibers and are no more able to produce proregenerating substances, such as GDNF, and become unable to maintain important trophic support for both motor and sensory neurons. ${ }^{37-39}$ This phenomenon exists even if a fresh graft is interposed between the proximal and distal nerve stump. As a consequence, the regenerating nerve fibers decrease in quantity and are much less myelinated. ${ }^{6}$ Thus, the exogenous supply of trophic substances improves the ability of Schwann cells to play their role, which improves the peripheral nerve regeneration in the chronically denervated distal nerve stump. ${ }^{40}$ This phenomenon is confirmed by the maximum changes of histological pattern in the distal nerve stump and the larger number of retrogradely labeled motoneuron in the facial nucleus in the GDNF channel subgroup.

The animals of the delayed graft group could also be considered as aged animals compared to the immediate repair group. This was confirmed by the histological results obtained in the two different groups. The retrograde labeling showed a significantly larger number of labelled neurons in the unlesioned facial nucleus in the immediate group $(1,516 \pm 98$ labelled neurons $)$ than in the delayed group $(2,207 \pm 95$ labeled motoneurons, $P<$ .0003), due to the older age of the animals in the delayed group. The labeled neurons showed also less fluorescein in the immediate repair group than in the delayed repair group. Similarly, the number of myelinated axons was significantly larger in the unlesioned mandibular branch in the immediate repair group (1,404 \pm 36 myelinated fibers) than in the delayed group $(1,286 \pm 43$ myelinated fibers, $P<.05$ ). The GDNF could also have had a second effect in the delayed repair group by supporting the senescent Schwann cells, known to be less effective in the elderly $[5,41]$. 


\section{CONCLUSION}

In summary, GDNF is a potent molecule for promoting facial nerve regeneration after autologous nerve grafts in situations where the delay between the nerve injury and its repair is prolonged and by aged patients. On the contrary, GDNF should not be used in cases of immediate repair, as it seems to produce deleterious effects on peripheral nerve regeneration. The application of GDNF in the clinic to autologous nerve grafts could thus be limited to situations where the nerve graft is long (proximal lesions, cross-facial nerve grafting), and in cases of aged patients.

\section{Acknowledgment}

The authors thank Amgen, Inc. for the research material provided (GDNF) for the experiment, and the Novartis Foundation for its financial support.

\section{BIBLIOGRAPHY}

1. Spector JG. Neural repair in facial paralysis: clinical and experimental studies. Eur Arch Otorhinolaryngol 1997; 254(suppl 1):68-75.

2. Conley J, Baker DC, Selfe RW. Paralysis of the mandibular branch of the facial nerve. Plast Reconstr Surg 1982;70: $569-577$.

3. Kerrebijn JD, Freeman JL. Facial nerve reconstruction: outcome and failures. J Otolaryngol 1998;27:183-186.

4. Bertelli JA, dos Santos AR, Taleb M, Calixto JB, Mira JC, Ghizoni MF. Long interpositional nerve graft consistently induces incomplete motor and sensory recovery in the rat. An experimental model to test nerve repair. $J$ Neurosci Methods 2004;134:75-80.

5. Verdu E, Ceballos D, Vilches JJ, Navarro X. Influence of aging on peripheral nerve function and regeneration. J Peripher Nerv Syst 2000;5:191-208.

6. Sulaiman OA, Gordon T. Effects of short- and long-term Schwann cell denervation on peripheral nerve regeneration, myelination, and size. Glia 2000;32:234-246.

7. May M, Sobol SM, Mester SJ. Hypoglossal-facial nerve interpositional-jump graft for facial reanimation without tongue atrophy. Otolaryngol Head Neck Surg 1991;104: 818-825.

8. Galli SK, Valauri F, Komisar A. Facial reanimation by cross-facial nerve grafting: report of five cases. Ear Nose Throat $J$ 2002;81:25-29.

9. Fisch U. Cross-face grafting in facial paralysis. Arch Otolaryngol 1976;102:453-457.

10. Endo T, Hata J, Nakayama Y. Variations on the "babysitter" procedure for reconstruction of facial paralysis. $J$ Reconstr Microsurg 2000;16:37-43.

11. Terzis JK, Konofaos P. Nerve transfers in facial palsy. Facial Plast Surg 2008;24:177-193.

12. O'Brien BM, Pederson WC, Khazanchi RK, Morrison,WA, MacLoed AM, Kumar V. Results of management of facial palsy with microvascular free-muscle transfer. Plast Reconstr Surg 1990;86:12-24.

13. Huang EJ, Reichardt LF. Neurotrophins: roles in neuronal development and function. Annu Rev Neurosci 2001;24: 677-736.

14. Yan Q, Matheson C, Lopez OT. In vivo neurotrophic effects of GDNF on neonatal and adult facial motor neurons. Nature 1995;373:341-344.

15. Quartu M, Serra MP, Boi M, Ferretti MT, Lai ML, Del Fiacco M. Tissue distribution of Ret, GFRalpha-1, GFRalpha-2 and GFRalpha-3 receptors in the human brainstem at fetal, neonatal and adult age. Brain Res 2007;1173: $36-52$.
16. Parsadanian A, Pan Y, Li W, Myckatyn TM, Brakefield D. Astrocyte-derived transgene GDNF promotes complete and long-term survival of adult facial motoneurons following avulsion and differentially regulates the expression of transcription factors of AP-1 and ATF/CREB families. Exp Neurol 2006;200:26-37.

17. Burazin TC, Gundlach AL. Up-regulation of GDNFR-alpha and c-ret mRNA in facial motor neurons following facial nerve injury in the rat. Brain Res Mol Brain Res 1998; 55:331-336.

18. Hoke A, Cheng C, Zochodne DW. Expression of glial cell line-derived neurotrophic factor family of growth factors in peripheral nerve injury in rats. Neuroreport 2000;11: $1651-1654$.

19. Barras FM, Pasche P, Bouche N, Aebischer P, Zurn AD. Glial cell line-derived neurotrophic factor released by synthetic guidance channels promotes facial nerve regeneration in the rat. $J$ Neurosci Res 2002;70:746-755.

20. Fine EG, Decosterd I, Papaloizos M, Zurn AD, Aebischer P. GDNF and NGF released by synthetic guidance channels support sciatic nerve regeneration across a long gap. Eur $J$ Neurosci 2002;15:589-601.

21. Bloch J, Fine EG, Bouche N, Zurn AD, Aebischer P. Nerve growth factor- and neurotrophin-3-releasing guidance channels promote regeneration of the transected rat dorsal root. Exp Neurol 2001;172:425-432.

22. Hoffman D, Wahlberg L, Aebischer P. NGF released from a polymer matrix prevents loss of ChAT expression in basal forebrain neurons following a fimbria-fornix lesion. Exp Neurol 1990;110:39-44.

23. Conley J, Baker DC. The surgical treatment of extratemporal facial paralysis: an overview. Head Neck Surg 1978;1: $12-23$.

24. Anderl H. Cross-face nerve transplant. Clin Plast Surg 1979;6:433-449.

25. Spector JG, Lee P, Peterein J, Roufa D. Facial nerve regeneration through autologous nerve grafts: a clinical and experimental study. Laryngoscope 1991;101:537-554.

26. Gordon T, Sulaiman O, Boyd JG. Experimental strategies to promote functional recovery after peripheral nerve injuries. J Peripher Nerv Syst 2003;8:236-250.

27. Spector JG, Lee P, Derby A, Frierdich GE, Neises G, Roufa DG. Rabbit facial nerve regeneration in NGF-containing silastic tubes. Laryngoscope 1993;103:548-558.

28. Kohmura E, Yuguchi T, Yoshimine T, et al. BDNF atelocollagen mini-pellet accelerates facial nerve regeneration. Brain Res 1999;849:235-238.

29. Papaloizos MY, Holmquist B, Lundborg G. An experimental study of nerve grafting combined with silicone tubes in the rat model: functional outcome and specificity of muscle reinnervation. Restor Neurol Neurosci 1997;11: 161-168.

30. Buti M, Verdu E, Labrador RO, Vilches JJ, Fores J, Navarro X. Influence of physical parameters of nerve chambers on peripheral nerve regeneration and reinnervation. Exp Neurol 1996;137:26-33.

31. Lundborg G, Dahlin LB, Danielsen N, Gelberman RH, Longo FM, Powell HC, Varon S. Nerve regeneration in silicone chambers: influence of gap length and of distal stump components. Exp Neurol 1982;76:361-375.

32. Lundborg G, Dahlin LB, Danielsen N. Ulnar nerve repair by the silicone chamber technique. Case report. Scand $J$ Plast Reconstr Surg Hand Surg 1991;25:79-82.

33. Weber RA, Breidenbach WC, Brown RE, Jabaley ME, Mass DP. A randomized prospective study of polyglycolic acid conduits for digital nerve reconstruction in humans. Plast Reconstr Surg 2000;106:1036-1048.

34. Weber RA, Warner MR, Verheyden CN, Proctor WH. Functional evaluation of gap vs. abutment repair of peripheral nerves in the rat. $J$ Reconstr Microsurg 1996;12:159-163.

35. Heijke GC, Klopper PJ, Baljet B, van Doom IB. Silicone rubber tubulization in peripheral sensory nerve reconstruction: an experimental study in rabbits. Microsurgery 2001;21:306-316. 
36. Naveilhan P, ElShamy WM, Ernfors P. Differential regulation of mRNAs for GDNF and its receptors Ret and GDNFR alpha after sciatic nerve lesion in the mouse. Eur J Neurosci 1997;9:1450-1460.

37. Hoke A, Gordon T, Zochodne DW, Sulaiman OA. A decline in glial cell-line-derived neurotrophic factor expression is associated with impaired regeneration after long-term Schwann cell denervation. Exp Neurol 2002;173:77-85.

38. Frostick SP, Yin Q, Kemp GJ. Schwann cells, neurotrophic factors, and peripheral nerve regeneration. Microsurgery 1998;18:397-405.
39. McPhail LT, Oschipok LW, Lui J, Tetzlaff W. Both positive and negative factors regulate gene expression following chronic facial nerve resection. Exp Neurol 2005;195: 199-207.

40. Sulaiman OA, Gordon T. Transforming growth factor-beta and forskolin attenuate the adverse effects of long-term Schwann cell denervation on peripheral nerve regeneration in vivo. Glia 2002;37:206-218.

41. Vaughan DW. Effects of advancing age on peripheral nerve regeneration. J Comp Neurol 1992;323:219237. 